\title{
Beauty and the Beast: Superconformal Symmetry in a Monster Module
}

\author{
L. Dixon ${ }^{1, \star}$, P. Ginsparg ${ }^{2, \star \star}$ and J. Harvey ${ }^{3, \star \star \star}$ \\ 1,3 Physics Department, Princeton University, Princeton, NJ 08544, USA \\ 2 Lyman Laboratory of Physics, Harvard University, Cambridge, MA 02138, USA
}

\begin{abstract}
Frenkel, Lepowsky, and Meurman have constructed a representation of the largest sporadic simple finite group, the Fischer-Griess monster, as the automorphism group of the operator product algebra of a conformal field theory with central charge $c=24$. In string terminology, their construction corresponds to compactification on a $\mathbf{Z}_{2}$ asymmetric orbifold constructed from the torus $\mathbf{R}^{24} / \Lambda$, where $\Lambda$ is the Leech lattice. In this note we point out that their construction naturally embodies as well a larger algebraic structure, namely a super-Virasoro algebra with central charge $\hat{c}=16$, with the supersymmetry generator constructed in terms of bosonic twist fields.
\end{abstract}

\section{Introduction}

A fundamental example of the techniques of conformal field theory arises in the construction of a moonshine module for the Fischer-Griess monster (the largest sporadic simple finite group) by Frenkel, Lepowsky, and Meurman (FLM) [1]. While this construction explains some of the puzzling connections between the representation theory of the monster and modular forms (referred to as moonshine in [2]), many aspects of this connection remain obscure.

In this note we point out a further feature of the construction, namely the existence of an underlying superconformal algebra in which the fermionic component of the super stress-energy tensor is constructed in terms of bosonic twist fields. In Sects. 2 and 3, we will first present a brief summary of the Virasoro and super-Virasoro algebras and their representations in terms of operator product expansions. In Sects. 4 and 5, we then present a brief synopsis of the FLM construction of the monster in the language of conformal field theory. We have tried to make these sections particularly accessible to string theorists, who may wish to satisfy their cultural curiosity in the "monstrous game." In Sect. 6 we

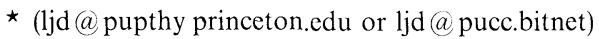

$\star \star$ (ginsparg@ huhepl.hepnet, ginsparg@ huhepl.bitnet, or ginsparg@ huhepl.harvard.edu)

$\star \star \star$ (jah@ pupthy.princeton.edu or jah (a pucc.bitnet) 
demonstrate the existence of a superconformal algebra in the construction, and show how the states in the theory are organized by the superconformal symmetry. In Sect. 7 we give a brief overview of the genus zero function field problem, an important open problem that links seemingly unrelated fields of mathematics. Finally in Sect. 8 we close with a discussion of issues related to those of this paper.

Our essential observation is that the $\mathbf{Z}_{2}$ twist operator for 24 bosonic fields has dimension $24 \cdot(1 / 16)=3 / 2$, where $1 / 16$ is the dimension of a single $\mathbf{Z}_{2}$ bosonic twist field. These dimension $3 / 2$ operators indeed prove to have all the correct properties to serve as supersymmetry generators for holomorphic conformal field theories based on $\mathbf{R}^{24} / \Lambda$ for any Niemeier lattice $\Lambda$, and in particular for the case $\Lambda=\Lambda_{\text {Leech }}$ used in the FLM construction. We hope that the additional algebraic structure revealed here will be useful in resolving some of the lingering mysterious features of the monster. We suspect also that the search for a unified understanding of all finite groups based on lattices, vertex operators, and loop algebras may be a mathematical arena where "physics" ideas such as supersymmetry will prove crucial.

\section{Conformal Field Theory}

In two-dimensional conformal field theory, analytic conformal transformations of the form $z \rightarrow z+\varepsilon z^{n+1}$ are generated by the moments

$$
L_{n}=\oint \frac{d z}{2 \pi i} z^{n+1} T(z)
$$

of the stress tensor $T(z)$. The $L_{n}$ 's satisfy the commutation relations

$$
\left[L_{m}, L_{n}\right]=(m-n) L_{m+n}+\frac{c}{12}\left(m^{3}-m\right) \delta_{m+n, 0}
$$

of the Virasoro algebra, where $c$ is a constant $\left(\left[L_{n}, c\right]=0\right)$ known as the central charge of the algebra. A general conformal field theory will involve as well anti-analytic conformal transformations generated by $\bar{L}_{n}$ 's. The commutation relations (2.2) are equivalent to the operator product expansion (OPE)

$$
T\left(z_{1}\right) T\left(z_{2}\right) \sim \frac{c / 2}{\left(z_{1}-z_{2}\right)^{4}}+\frac{2}{\left(z_{1}-z_{2}\right)^{2}} T\left(z_{2}\right)+\frac{1}{\left(z_{1}-z_{2}\right)} \partial_{2} T\left(z_{2}\right)+\cdots,
$$

where we shall systematically suppress the non-singular terms in what follows. The equivalence between (2.2) and (2.3) follows from standard contour deformation arguments. (For introductions to standard conformal field theory techniques, see e.g. [3].)

Fields that satisfy the OPE

$$
T\left(z_{1}\right) \phi\left(z_{2}\right) \sim \frac{h}{\left(z_{1}-z_{2}\right)^{2}} \phi\left(z_{2}\right)+\frac{1}{\left(z_{1}-z_{2}\right)} \partial_{2} \phi\left(z_{2}\right)+\cdots
$$

are termed primary fields of dimension, or conformal weight, $h$. Each primary field is associated with a highest weight state $|h\rangle=\phi(0)|0\rangle$, where $|0\rangle$ is the 
vacuum state (associated with the identity operator). Equation (2.4) implies that $L_{0}|h\rangle=h|h\rangle$ and $L_{n}|h\rangle=0, n>0$. Applying the "creation" operators $L_{n}, n<0$, to any highest weight $|h\rangle$ gives a (reducible) representation of the Virasoro algebra called a Verma Module, which we denote $\mathbf{M}_{h, c}$. This module has an integer grading $\mathbf{M}_{h, c}=\oplus_{n} \mathbf{M}_{h, c}^{n}$, where $n$ is the eigenvalue of $L_{0}-h$, i.e.

$$
\mathbf{M}_{h, c}^{n}=\left\{v \in \mathbf{M}_{h, c} \mid L_{0} v=(h+n) v\right\} .
$$

Its character is given by

$$
\operatorname{ch} \mathbf{M}_{h, c}(q) \equiv q^{h-c / 24} \sum_{n=0}^{\infty}\left(\operatorname{dim} \mathbf{M}_{h, c}^{n}\right) q^{n}=\frac{q^{h-c / 24}}{\prod_{n=1}^{\infty}\left(1-q^{n}\right)}
$$

From a physical point of view, the factor of $q^{-c / 24}$ introduced in (2.6) arises as a vacuum energy; from a mathematical point of view, it improves the modular transformation properties of the partition function (to be discussed below).

Unitarity of representations of the Virasoro algebra in general restricts the values of $h$ and $c$ [4]. In many cases the Verma module contains null states, which means that it provides a reducible representation of the Virasoro algebra, and one must factor out the null states to obtain an irreducible module, denoted by $\mathbf{L}_{h, c}$. When $c>1$, however, all representations with $h \geqq 0$ are unitary, and all Verma modules with $h>0$ are irreducible, so we have

$$
\begin{aligned}
\operatorname{ch} \mathbf{L}_{h, c}(q) & =\operatorname{ch} \mathbf{M}_{h, c}(q), \quad c>1, \quad h>0, \\
\operatorname{ch} \mathbf{L}_{0, c}(q) & =\frac{q^{-c / 24}}{\prod_{n=2}^{\infty}\left(1-q^{n}\right)} .
\end{aligned}
$$

In a general conformal field theory, highest weight states are labelled by their conformal weights $(h, \bar{h})$, i.e. their eigenvalues under $L_{0}$ and $\bar{L}_{0}$ respectively. The genus one partition function of the theory is given by

$$
Z_{c}(q, \bar{q})=\sum_{h, \bar{h}} N_{h, \bar{h}} \operatorname{ch} \mathbf{L}_{h, c}(q) \operatorname{ch} \mathbf{L}_{\bar{h}, c}(\bar{q}),
$$

where $N_{h, \bar{h}}$ is an integer equal to the number of primary fields with conformal weight $(h, \bar{h})$. Moreover if we write $q=\exp (2 \pi i \tau)$, where $\tau$ is the modular parameter for a torus, then $Z_{c}(q, \bar{q})$ should be invariant under modular transformations

$$
\tau \rightarrow \frac{a \tau+b}{c \tau+\frac{1}{d}}, \quad\left(\begin{array}{ll}
a & b \\
c & d
\end{array}\right) \in S L(2, \mathbf{Z}) .
$$

For $c>1$ this requires that the number of primary fields be infinite [5]. In certain cases, $Z_{c}(q, \bar{q})$ will factorize into holomorphic and anti-holomorphic parts, both of which are modular covariant, and the anti-holomorphic part may be ignored. (Even in this case, however, generalization of the partition function to higher genus Riemann surfaces may require inclusion of both holomorphic and antiholomorphic parts [6].) In what follows we shall concentrate exclusively on the case of purely holomorphic conformal field theories. 


\section{Superconformal Field Theory}

Supersymmetric extensions of the Virasoro algebra are obtained by generalizing conformal transformations to superconformal transformations of supercoordinates $\mathbf{z}=(z, \theta)$, where $\theta$ is an anticommuting coordinate $\left(\theta^{2}=0\right)$. Superconformal transformations are generated by the moments of a super stress-energy tensor. If there is only a single anti-commuting coordinate ( $N=1$ supersymmetry) then the super stress-energy tensor $\mathbf{T}(\mathbf{z})=T_{F}(z)+\theta T_{B}(z)$ has components that satisfy the operator products

$$
\begin{aligned}
& T_{B}\left(z_{1}\right) T_{B}\left(z_{2}\right) \sim \frac{3 \hat{c} / 4}{\left(z_{1}-z_{2}\right)^{4}}+\frac{2}{\left(z_{1}-z_{2}\right)^{2}} T_{B}\left(z_{2}\right)+\frac{1}{\left(z_{1}-z_{2}\right)} \partial_{2} T_{B}\left(z_{2}\right), \\
& T_{B}\left(z_{1}\right) T_{F}\left(z_{2}\right) \sim \frac{3 / 2}{\left(z_{1}-z_{2}\right)^{2}} T_{F}\left(z_{2}\right)+\frac{1}{\left(z_{1}-z_{2}\right)} \partial_{2} T_{F}\left(z_{2}\right), \\
& T_{F}\left(z_{1}\right) T_{F}\left(z_{2}\right) \sim \frac{\hat{c} / 4}{\left(z_{1}-z_{2}\right)^{3}}+\frac{1 / 2}{\left(z_{1}-z_{2}\right)} T_{B}\left(z_{2}\right),
\end{aligned}
$$

where $\hat{c}=\frac{2}{3} c$. The conventional normalization is such that the stress-energy tensor for a single free bosonic field $x(z)$ has central charge $c=1$ in (2.3), and that for a single free superfield $x(z)+\theta \psi(z)$ has central charge $\hat{c}=1$ in (3.1). The second equation in (3.1) is the statement that $T_{F}$ is a primary field of dimension $3 / 2$.

In terms of the moments $L_{n}$ of $T_{B}$, and the moments

$$
G_{n}=\oint \frac{d z}{2 \pi i} z^{n+1 / 2} 2 T_{F}(z)
$$

of $T_{F}$, the OPE's (3.1) are equivalent to the (anti-)commutation relations

$$
\begin{aligned}
& {\left[L_{m}, L_{n}\right]=(m-n) L_{m+n}+\frac{\hat{c}}{8}\left(m^{3}-m\right) \delta_{m+n, 0},} \\
& {\left[L_{m}, G_{n}\right]=\left(\frac{m}{2}-n\right) G_{m+n},} \\
& \left\{G_{m}, G_{n}\right\}=2 L_{m+n}+\frac{\hat{c}}{2}\left(m^{2}-\frac{1}{4}\right) \delta_{m+n, 0} .
\end{aligned}
$$

For integer moding $(n \in \mathbf{Z})$ of $G_{n}$, the supersymmetric extension of the Virasoro algebra is termed the Ramond (R) algebra; for half-integer moding $\left(n \in \mathbf{Z}+\frac{1}{2}\right)$, it is termed the Neveu-Schwarz (NS) algebra. Primary fields are again associated with highest weight states $|h\rangle$, satisfying $\left.L_{n}|h\rangle=G_{n}|h\rangle=0, n\right\rangle 0$, and $L_{0}|h\rangle=h|h\rangle$. Note that (3.3) requires that a highest weight state in the Ramond sector have eigenvalue $h-\hat{c} / 16$ under $G_{0}^{2}$. For $\hat{c}>1$, the only restrictions imposed by unitarity are $h \geqq 0(\mathrm{NS})$, and $h \geqq \hat{c} / 16(\mathrm{R})$, and the Verma modules again provide irreducible representations unless the latter inequalities are saturated.

In order to obtain a modular invariant partition function, it is necessary to include representations of both the R and NS algebras together with a projection 
onto a subset of the highest weight states [7]. This is accomplished by introducing an operator $\hat{\Gamma}$ that commutes with $T_{B}$ and $\bar{T}_{B}$, anticommutes with $T_{F}$ and $\bar{T}_{F}$, and satisfies $\hat{\Gamma}^{2}=1$. The modular invariant partition function then takes the form

$$
Z(q, \bar{q})=(q \bar{q})^{-\hat{c} / 16}\left(\operatorname{Tr}_{\mathrm{NS}} P q^{L_{0}} \bar{q}^{\bar{L}_{0}}+\operatorname{Tr}_{\mathrm{R}} P q^{L_{0}} \bar{q}^{\bar{L}_{0}}\right),
$$

where the projection $P=(1+\hat{\Gamma}) / 2$. In the Ramond algebra, $G_{0}$ commutes with $L_{0}$ and satisfies $G_{0}^{2}=L_{0}-\hat{c} / 16$. Thus if $h>\hat{c} / 16$, highest weight states of the Virasoro algebra are paired by $G_{0}$ with states having opposite eigenvalue under $\hat{\Gamma}$. Only the Ramond ground states, with $h=\hat{c} / 16$, are not so paired since they may be annihilated by $G_{0}$. It follows that the contribution

$$
\pm(q \bar{q})^{-\hat{c} / 16} \operatorname{Tr}_{\mathrm{R}} \hat{\Gamma} q^{L_{0}} \bar{q}^{\bar{L}_{0}}
$$

to the partition function is always a constant (analogous to the Witten index [8]). The value of this constant is not determined by genus-one modular invariance, but rather by the requirement of a consistent operator interpretation of the terms in (3.4). If a nonzero value of the constant is permitted, the two sign choices in (3.5) generate inequivalent modular invariant theories $[9,10]$, each consistent, and distinguished from one another by an opposite choice of projection in the Ramond sector.

It is not always obvious whether a given conformal field theory has a superconformal symmetry. One problem is that the state with conformal weight $\left(\frac{3}{2}, 0\right)$ created by $T_{F}$ itself cannot survive in a modular invariant theory, since invariance under $\tau \rightarrow \tau+1$ allows only integer spin $s=h-\bar{h}$ for all physical states. Before projection on $\hat{\Gamma}=+1$ states however, the $\hat{\Gamma}=-1$ states of the theory will include in general states with half-integer spin. Projection by $P$ removes all these states and leaves a modular invariant local theory. To identify a superconformal algebra, one must therefore look beyond the physical states and construct a non-local $\mathbf{Z}_{2}$ cover of the theory containing a weight $\left(\frac{3}{2}, 0\right)$ primary field that satisfies the OPE (3.1). The $\mathbf{Z}_{2}$ cover will consist of the set of all fields in the original conformal field theory, plus $T_{F}$, plus any fields needed to close the operator algebra of $T_{F}$ with the original fields. All OPE's in the covering theory are either local (i.e. have integer power singularities), or have at worst square-root type singularities. Finally, there is a projection onto $\mathbf{Z}_{2}$ invariant operators of some $\mathbf{Z}_{2}$ symmetry that yields the original local conformal field theory. (A general method for constructing this non-local $\mathbf{Z}_{2}$ cover will be outlined at the end of Sect. 6. It makes use of the $\mathbf{Z}_{2}$ symmetry-possessed by any superconformal field theory-that acts as +1 and -1 on Neveu-Schwarz and Ramond sectors respectively.)

Conversely, given a local theory with a non-local $\mathbf{Z}_{2}$ cover that contains a dimension $\left(\frac{3}{2}, 0\right)$ field satisfying (3.1), one can always organize the states into representations of a superconformal algebra with the $\left(\frac{3}{2}, 0\right)$ field playing the role of $T_{F}$. Fields in the Ramond sector will be those with square-root singularities in their OPE with $T_{F}$, while those in the NS sector will have integer power singularities. The intent here is to show that the holomorphic conformal field theory used in the FLM construction of the monster possesses precisely this structure. 


\section{Lattices and Holomorphic Conformal Field Theory}

Given an even, self-dual, integer lattice $\Lambda$ of dimension $r$, we can always define a holomorphic modular-covariant conformal field theory with central charge $c=r$. First we introduce $r$ free holomorphic fields $x^{\mathrm{i}}(z), i=1, \ldots, r$, that we view as coordinates on the torus $\mathbf{T}^{r}=\mathbf{R}^{r} / \Lambda$, i.e. $x \equiv x+2 \pi \beta, \beta \in \Lambda$. The operators in their mode expansions ${ }^{1}$

$$
x^{i}(z)=q^{i}-i p^{i} \ln z+i \sum_{n \neq 0} \frac{\alpha_{n}^{i}}{n} z^{-n}
$$

satisfy the commutation relations

$$
\left[q^{i}, p^{j}\right]=-i \delta^{i j}, \quad\left[\alpha_{n}^{i}, \alpha_{m}^{j}\right]=n \delta^{i j} \delta_{n+m, 0} .
$$

For every lattice vector $\beta \in \Lambda$, we can construct a Fock space $F_{\beta}$. It is built on the highest weight state $|\beta\rangle$, which satisfies $\alpha_{n}|\beta\rangle=0$ for $n>0$ and $p^{i}|\beta\rangle=\beta^{i}|\beta\rangle$ ( $\beta^{i}$ are the components of $\beta$ in an orthonormal frame). States of these Fock spaces are constructed by acting on $|\beta\rangle$ with the creation operators $\alpha_{-n}, n>0$.

A holomorphic conformal field theory may be defined via a field theory action as follows. We begin with the action

$$
S=\frac{1}{2 \pi} \int \partial X^{i} \bar{\partial} X^{i}+B_{i j} \partial X^{i} \bar{\partial} X^{j}=\frac{1}{2 \pi} \int g_{a b} \partial X^{a} \bar{\partial} X^{b}+b_{a b} \partial X^{a} \bar{\partial} X^{b},
$$

where the fields $X^{i}(z, \bar{z}) \in \mathbf{R}^{r} /(\Lambda / 2)$, i.e. $X^{i} \equiv X^{i}+2 \pi\left(\beta^{i} / 2\right), \beta \in \Lambda$. We take the even, self-dual, lattice $\Lambda$ of dimension $r$ to be generated by basis vectors $e_{a}, a=1, \ldots, r$, so that $\beta=n^{a} e_{a}, n^{a} \in \mathbf{Z}$, and define a metric $g_{a b}=e_{a} \cdot e_{b}$. The coordinates $X^{a}$ in (4.3) are defined by $X^{i}=\left(e_{a}\right)^{i} X^{a}$, and are thus periodic up to multiples of $\pi, X^{a} \equiv$ $X^{a}+2 \pi\left(n^{a} / 2\right)$. Note that the vectors $e^{* a}=g^{a b} e_{b}$, defined in terms of the inverse metric $g^{a b}=g_{a b}^{-1}$, satisfy $e^{* a} \cdot e_{b}=\delta_{b}^{a}$, and by self-duality are consequently also a basis of $\Lambda$. Finally $b_{a b}=e_{a} \cdot B \cdot e_{b}=B_{i j}\left(e_{a}\right)^{i}\left(e_{b}\right)^{j}$ are the components of the (constant) antisymmetric $B$-field referred to lattice frames.

The partition function for the theory (4.3) is [11]

$$
Z(q, \bar{q})=(q \bar{q})^{-r / 24} \operatorname{Tr} q^{L_{0}} \bar{q}^{\bar{L}_{0}}=\frac{1}{(\eta \bar{\eta})^{r}} \sum_{w \in \Lambda / 2, p \in 2 \Lambda} q^{(1 / 2) p_{L}^{2}} \bar{q}^{(1 / 2) p_{R}^{2}}
$$

where $\eta(q)=q^{1 / 24} \prod_{n=1}^{\infty}\left(1-q^{n}\right)$ is the Dedekind eta function, and $p_{L, R}=\frac{1}{2} p-B w \pm$ $w=m_{a} e^{* a}-\frac{1}{2}\left(b_{a b} \stackrel{n=1}{\mp} g_{a b}\right) e^{* a} n^{b}$. In general the summations in (4.4) are not consistent with a splitting between holomorphic and anti-holomorphic Hilbert spaces. But if the $B$-field is chosen to satisfy

$$
e_{a} \cdot B \cdot e_{b}=e_{a} \cdot e_{b} \bmod 2
$$

(e.g. take $b_{a b}= \pm g_{a b}, a \lessgtr b$ ), the summation in (4.4) is easily seen to decompose into independent summations over $p_{L} \in \Lambda$ and $p_{R} \in \Lambda$. Equation (4.4) then takes the

\footnotetext{
1 Our normalization conventions are such that the operator products satisfy $\partial x^{i}(z) \partial x^{j}(w) \sim-\delta^{i j} /(z-w)^{2}$
} 
factorized form

$$
Z(q, \bar{q})=\left|\frac{1}{\eta^{r}} \sum_{\beta \in \Lambda} q^{(1 / 2) \beta^{2}}\right|^{2}
$$

(and we would have e.g. the factorization $N_{h, \bar{h}}=N_{h} N_{\bar{h}}$ in (2.8)).

In the case at hand, one can show as well that the Hilbert space decomposes into a product of holomorphic and anti-holomorphic pieces and one can therefore impose a consistent projection onto the purely holomorphic part. This is the theory that we shall consider in what follows, i.e. we shall retain only the dependence on the coordinate $x(z)$, where $X(z, \bar{z})=\frac{1}{2}(x(z)+x(\bar{z})$ ). (This defines what we mean by holomorphic conformal field theory on $\mathbf{R}^{r} / \Lambda$, although strictly speaking we should probably call it something like $\mathbf{R}^{r} /(2 \pi \Lambda)^{1 / 2}$.)

The stress-energy tensor of the theory is defined as

$$
T(z)=-\frac{1}{2}: \partial_{z} x \cdot \partial_{z} x
$$

(where the double dots indicate normal ordering), with the corresponding Virasoro generators given by

$$
L_{n}=\frac{1}{2} \sum_{m=-\infty}^{m=+\infty} \alpha_{n-m} \cdot \alpha_{m}, \quad n \neq 0
$$

where $\alpha_{0}^{i}=p^{i}$. To define $L_{0}$ we must resolve the operator ordering ambiguity that arises due to the non-commutativity of $\alpha_{n}$ and $\alpha_{-n}$. The definition

$$
L_{0}=\frac{1}{2} p^{2}+\sum_{n=1}^{\infty} \alpha_{-n} \cdot \alpha_{n},
$$

with the vacuum normal ordering constant instead included in the prefactor to the partition function, satisfies the commutation relations (2.2).

Primary fields are given by particular products of the dimension one $U(1)$ currents $j^{i}(z)=i \partial_{z} x^{i}$ and the dimension $\beta^{2} / 2$ exponentials

$$
V_{\beta}(z)=: e^{i \beta \cdot x(z)}, \quad \beta \in \Lambda .
$$

We see that the vertex operators $V_{\beta}$ are well defined under $x \rightarrow x+2 \pi \alpha, \alpha \in \Lambda$, since then $\beta \cdot \alpha \in \mathbf{Z}$. Strictly speaking, (4.9) is incorrect because the OPE of these fields is not associative. Associativity of the OPE is restored by constructing [12] a projective representation $\bar{\Lambda}$ of the lattice $\Lambda$ with two-cocyle $\varepsilon(\alpha, \beta)$, and including on the right-hand side of (4.9) a factor $c(\alpha)$ that satisfies $c(\alpha) c(\beta)=\varepsilon(\alpha, \beta) c(\alpha+\beta)$. This projective representation for the Leech lattice is described in detail by FLM. In terms of the antisymmetric $B$ field mentioned above, we note that we can represent $\varepsilon(\alpha, \beta)=e^{i \pi \alpha \cdot B \cdot \beta / 2}$. It then follows from (4.5) that $\varepsilon(\alpha, \beta) \varepsilon^{-1}(\beta, \alpha)=$ $(-1)^{\alpha \cdot B \cdot \beta}=(-1)^{\alpha \cdot \beta}$, as required for associativity of the OPE's of the $c(\alpha): e^{i \alpha \cdot x(z)}:$ 's. (In terms of the momentum operator $p$, the $c(\alpha)$ 's themselves can be represented in this case as $c(\alpha)=e^{i \pi \alpha \cdot B \cdot p / 2}$.)

The partition function for the theory is given by

$$
Z(q)=q^{-c / 24} \operatorname{Tr} q^{L_{0}}=\frac{\Theta_{\Lambda}(q)}{\eta^{r}(q)},
$$


where $\Theta_{\Lambda}(q)=\sum_{\beta \in \Lambda} q^{(1 / 2) \beta^{2}}$ is the theta function of the lattice $\Lambda$. For $c$ a multiple of $24, Z(q)$ will be modular invariant; for $c=8,16 \bmod 24$, on the other hand, $Z(q)$ will be modular covariant, i.e. a weight-zero modular function with non-trivial multiplier (in other words for the cases above it picks up a phase $e^{2 \pi i c / 24}$ under the modular transformation $\tau \rightarrow \tau+1$ ). An example of a holomorphic conformal field theory arises for the choice $\Lambda=\Gamma_{8}$, where $\Gamma_{8}$ is the root lattice of $E_{8}$. The holomorphic partition function is then the character of the (basic) representation of the level one affine Kac-Moody algebra $\hat{E}_{8}$. It is thus given by $\Theta_{\Gamma_{8}} / \eta^{8}=j^{1 / 3}$, where the famous weight-zero modular function $j$ is given by $j(q)=q^{-1}+744+$ $196884 q+\cdots$.

In what follows we shall ultimately be interested in the case $\Lambda=\Lambda_{\text {Leech }}$, where the Leech lattice $\Lambda_{\text {Leech }}$ is the unique dimension 24 , even, self-dual lattice with $\Lambda_{2}=\emptyset\left(\Lambda_{n}\right.$ is the set of points in $\Lambda$ with length-squared $\left.n\right)$. For this case, the holomorphic conformal field theory constructed by the above procedure has partition function

$$
Z_{\text {Leech }}(q)=\frac{\Theta_{\Lambda_{\text {Lecch }}}}{\eta^{24}}=\frac{1}{q}+24+196884 q+\cdots=J(q)+24,
$$

with $J(q)=j(q)-744$ (i.e. $J$ has constant term set equal to zero). For $c=24$ we see that the partition function is indeed modular invariant. Part of monstrous moonshine is the fact that the terms in the expansion (4.11), apart from the constant term, are simple sums of the dimensions of irreducible representations of the monster. In the next section we shall review how the FLM construction removes the unwanted constant term.

Given a modular invariant conformal field theory with a discrete symmetry group $G$, it is often possible to construct a new conformal field theory by the procedure of "modding out" or "twisting" by $G$ [13]. This procedure can be applied to holomorphic conformal field theories based on even, self-dual lattices $A$, and modular invariance puts stringent restrictions on $G$. In the simplest case $G$ will be a $\mathbf{Z}_{n}$ automorphism of $\Lambda$, generated by the $S O(r)$ rotation (in a diagonal basis)

$$
g=\operatorname{diag}\left(e^{2 \pi i \xi_{1} / n}, \ldots, e^{2 \pi i \xi_{l} / n} ; e^{-2 \pi i \xi_{1} / n}, \ldots, e^{-2 \pi i \xi_{l} / n}\right),
$$

where $l=r / 2$. Then the restriction on $G$ is $[13,14]$

$$
\sum_{i=1}^{l} \xi_{i}^{2}=0\left\{\begin{array}{ll}
\bmod n & n \text { odd } \\
\bmod 2 n & n \text { even }
\end{array} .\right.
$$

For $n$ even, there is an additional restriction particular to asymmetric bosonic orbifolds $[15,16]$,

$$
\beta \cdot g^{n / 2} \beta=0 \bmod 2, \quad \text { for all } \beta \in \Lambda \text {. }
$$

Since even, self-dual, lattices exist only in $8 k$ dimensions, a twist by an involutive automorphism $g$ defined to take $x \rightarrow-x$, and hence lattice vectors $\beta \rightarrow-\beta$, will always satisfy (4.13)-(4.14). This is because $-\beta^{2}=0 \bmod 2$, and we have $n=2$ and all the $\xi_{i}=1$ in (4.12), giving $\sum \xi_{i}^{2}=l=4 k$. In the same way that we can view the conformal field theory determined by $\Lambda$ as corresponding to string compactification 
on the torus $\mathbf{T}^{r}=\mathbf{R}^{r} / \Lambda$, we can view the twisting procedure as corresponding to string compactification on the orbifold $\mathbf{T}^{r} / \mathbf{Z}_{2}$. Note that the $\mathbf{Z}_{2}$ twists that we consider here have the simplifying feature that they leave no sublattice of $\Lambda$ invariant, so the fixed points are classified by $\Lambda / 2 \Lambda$.

To implement the twisting procedure for $\mathbf{Z}_{2}$ twists, we first construct the untwisted Hilbert space $H_{(+)}$as a direct product of the Fock spaces $F_{\beta} . H_{(+)}$has a $\mathbf{Z}_{2}$ grading induced by the action of the generator $g$ of $G=\mathbf{Z}_{2}$, and we define

$$
H_{(+)}^{ \pm}=\left\{v \in H_{(+)} \mid g v= \pm v\right\} \text {. }
$$

To construct a twisted Hilbert space $H_{(-)}$, we take fields $x^{i}(z)$ satisfying boundary conditions $x^{i}\left(e^{2 \pi i} z\right)=-x^{i}(z)(\bmod \Lambda)$ with mode expansion

$$
x^{i}(z)=\tilde{q}^{i}+i \sum_{n \in Z+1 / 2} \frac{\alpha_{n}^{i}}{n} z^{-n},
$$

where $\alpha_{n}^{i}$ obey the same commutation relations (4.2) as before.

For a $\mathbf{Z}_{2}$ twist acting on both holomorphic and anti-holomorphic parts of $X(z, \bar{z})=\frac{1}{2}(x(z)+x(\bar{z})), \tilde{q}^{i}$ would take values in the $2^{r}$ dimensional fixed-point set of $g$, given by $\Lambda / 2 \Lambda$. Because the twist acts only on the holomorphic field $x(z)$, the situation is more subtle $[1,15,16]$. The two-cocycle $\varepsilon(\alpha, \beta)$ determines a $\mathbf{Z}_{2}$ central extension of $\Lambda$, and also a $\mathbf{Z}_{2}$ extension of $\Lambda / 2 \Lambda=\left(\mathbf{Z}_{2}\right)^{24}$ (viewing the lattice $\Lambda$ as an abelian group). This central extension of $\Lambda / 2 \Lambda$ has a $2^{12}$ dimensional irreducible representation, acting on a space $T$ with basis elements $\left|\sigma^{a}\right\rangle=\sigma^{a}(0)|0\rangle$, $a=0,1, \ldots, 2^{12}-1$. The $\left|\sigma^{a}\right\rangle$ are particular linear combinations of the $2^{24}$ states, denoted $\left|\tilde{q}_{b}\right\rangle$, with definite values of $\tilde{q}$ :

$$
\tilde{q}\left|\tilde{q}_{b}\right\rangle=\tilde{q}_{b}\left|\tilde{q}_{b}\right\rangle, \text { where } 2 \tilde{q}_{b} \in \Lambda / 2 \Lambda \text {. }
$$

(The factor $2^{12}$ can also be regarded as the square root of the number of fixed points of the transformation $X^{i}(z, \bar{z}) \rightarrow-X^{i}(z, \bar{z})$. The $2^{24}$ fixed points transform as a $\left(2^{12}, 2^{12}\right)$ representation of the holomorphic and anti-holomorphic symmetry groups. The projection onto the holomorphic Hilbert space removes the right $2^{12}$ degeneracy.) The twisted Hilbert space is then constructed as the tensor product

$$
H_{(-)}=T \otimes F_{(-)},
$$

where the twisted Fock space $F_{(-)}$is constructed by acting with the $\alpha_{-n}, n>0$ $\left(n \in \mathbf{Z}+\frac{1}{2}\right)$, on the Fock vacuum. $H_{(-)}$also has a $\mathbf{Z}_{2}$ grading induced by the action of $g$ as in (4.15), and we refer to the corresponding eigenspaces as $H_{(-)}^{ \pm}$.

Using (4.6) and the mode expansion (4.16) gives for the Virasoro generators acting in the twisted Hilbert space

$$
\begin{aligned}
& L_{n}=\frac{1}{2} \sum_{m \in Z+1 / 2} \alpha_{n-m} \cdot \alpha_{m}, \quad n \neq 0, \\
& L_{0}=\sum_{m=1 / 2}^{\infty} \alpha_{-m} \cdot \alpha_{m}+\frac{r}{16} .
\end{aligned}
$$

The vacuum normal ordering constant $r / 16$ for $L_{0}$ in the twisted sector (relative to the untwisted vacuum) is required in order that (4.19) satisfies the Virasoro 
algebra (2.2) (as can be seen using $\left.\frac{1}{2} \sum_{n=0}^{\infty}\left(n+\frac{1}{2}\right)=-\frac{1}{4} \zeta(-1)=\frac{1}{48}=-\frac{1}{24}+\frac{1}{16}\right)$. (An alternate derivation of the constant will appear shortly.)

The full partition function of the twisted theory is given by

$$
\begin{aligned}
Z_{\mathrm{tw}}(q) & =q^{-r / 24}\left(\operatorname{Tr}_{H_{(+)}^{+}} q^{L_{0}}+\operatorname{Tr}_{H_{(-)}^{+}} q^{L_{0}}\right) \\
& =q^{-r / 24}\left(\operatorname{Tr}_{H_{(+)}} P q^{L_{0}}+\operatorname{Tr}_{H_{(-)}} P q^{L_{0}}\right),
\end{aligned}
$$

where the traces run over the indicated Hilbert spaces, and $P=(1+g) / 2$. An intuitive understanding of the modular properties of the terms appearing in $Z_{\mathrm{tw}}$ comes from the path integral interpretation of the traces appearing in (4.20). A term of the form $\operatorname{Tr}_{H_{h}} g q^{L_{0}}$ evaluated in the Hilbert space $H_{h}$ twisted by the group element $h$, denoted by

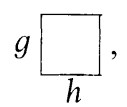

can be computed as a path integral over a torus with modular parameter $\tau$ and boundary conditions twisted by $g$ and $h$ in the "time" and "space" directions respectively (in (4.20) we have $H_{(+)} \equiv H_{1}$ and $H_{(-)} \equiv H_{g}$ ). A modular transformation $\tau \rightarrow(a \tau+b) /(c \tau+d)$ acts as a global diffeomorphism of the torus and changes the boundary conditions to

$Z_{\mathrm{tw}}$ in (4.20) is given by the sum

$$
g^{a} h^{b} \underset{g^{c} h^{d}}{\square} \text {. }
$$

$$
Z_{\mathrm{tw}}=\frac{1}{2}\left(1 \underset{1}{\square_{1}}+g \square_{1}+1 \square_{g}+g \underset{ }{\square}\right),
$$

which is modular invariant provided that (4.13) and (4.14) are satisfied.

In conformal field theory the $2^{r / 2}$ degenerate twisted ground states belonging to $T$ are created from the untwisted ground state by primary conformal fields known as twist fields, and denoted by $\sigma^{a}$. Because the $\sigma^{a}$ change the sign of $\partial_{z} x$, they have the non-local OPE

$$
\partial x^{i}(z) \sigma^{a}(w) \sim(z-w)^{-1 / 2} \tau^{i a}(w)+\cdots,
$$

where $\tau$ is an excited twist field of dimension 2 that creates excited states in $H_{(-)}$. The projection onto states in $H_{(+)}^{+}$and $H_{(-)}^{+}$keeps a set of operators that have mutually local OPE's.

Using the OPE (4.24), one can derive the conformal dimension of the fields $\sigma^{a}$, which is also the normal-ordering constant for the vacuum in the twisted sector. Consider the correlation function

$$
\frac{\left\langle 0\left|\sigma(\infty) \partial x^{i}(z) \partial x^{j}(w) \sigma(0)\right| 0\right\rangle}{\langle 0|\sigma(\infty) \sigma(0)| 0\rangle}=-\frac{\frac{1}{2}\left(\sqrt{\frac{z}{w}}+\sqrt{\frac{w}{z}}\right) \delta^{i j}}{(z-w)^{2}}
$$

of bosonic fields in the twisted sector, where $\sigma$ is a twist field for all $r$ bosonic coordinates $x^{i}$. With the normal ordered stress-energy tensor $T(z)$ defined as $-\frac{1}{2}\left[\partial x(z) \cdot \partial x(w)+r /(z-w)^{2}\right]_{z \rightarrow w}$, we infer the leading operator product $T(z) \sigma(0) \sim$ 
$(r / 16) z^{-2} \sigma(0)+\cdots$, and hence from (2.4) we have $h=r / 16$. In a canonical normalization for the twist fields, their OPE's with each other are given by

$$
\sigma^{a}(z) \sigma^{b}(w) \sim \delta^{a b}(z-w)^{-r / 8}+\cdots .
$$

For the $c=8 E_{8}$ holomorphic conformal field theory, it is known that the above twisting procedure gives back an isomorphic theory. (In terms of the level one $\hat{D}_{8}$ subcontent, the projection in the untwisted sector removes the $\hat{D}_{8}$ spinor representation, leaving the character for the $\hat{D}_{8}$ basic representation; and the projection in the twisted sector removes the $\hat{D}_{8}$ vector, leaving the character for the other $\hat{D}_{8}$ spinor [1]). In the $c=16$ case, it is known $[13,17]$ that this twisting procedure simply interchanges the two holomorphic conformal field theories based on the $E_{8} \times E_{8}$ and $\operatorname{Spin}(32) / \mathbf{Z}_{2}$ lattices. The first case for which the procedure produces new interesting holomorphic conformal field theories occurs at $c=24$, and the most interesting of these comes from modding out the conformal field theory based on the Leech lattice.

\section{The Moonshine Module}

In the construction of the monster, the need for the $\mathbf{Z}_{2}$ twisting described above is motivated by finite group theory [1]. The conformal field theory determined by the Leech lattice has a large symmetry group that acts as an automorphism group of the operator product algebra. Naively, one would expect this to be just the automorphism group 0 of the Leech lattice, which is also known as the Conway group. Due to the cocycle factors that appear in the definition of the vertex operators, however, the automorphism group is actually an extension of $\cdot 0$ by the abelian group $\left(\mathbf{Z}_{2}\right)^{24}$, denoted by $2^{24}(\cdot 0)$. This group has an analog in the theory of Lie algebras of type A,D,E. There one finds that the order two reflections in simple roots that generate the Weyl group $W$ lift to automorphisms of the Lie algebra which are of order four. This yields an automorphism group of the Lie algebra that is an extension of $W$ by $\left(\mathbf{Z}_{2}\right)^{r}$, where $r$ is the rank of the corresponding root lattice [18].

The group $2^{24}(\cdot 0)$ is very similar in structure to the centralizer of an involution that appears in the monster. (An involution, $i$, is an element of order two; its centralizer in the monster consists of all elements $g$ of the monster for which $g^{-1} i g=i$.) This centralizer, denoted by $C=2_{+}^{1+24}(\cdot 1)$, is given by an extension of $\cdot 1=\cdot 0 / \mathbf{Z}_{2}$ (the simple Conway group) by an "extra-special" group denoted $2_{+}^{1+24}$, the latter having a $2^{12}$-dimensional irreducible representation. The group $2_{+}^{1+24}$ is isomorphic to the $Z_{2}$ central extension of $\Lambda / 2 \Lambda$ discussed previously. The extra-special groups that arise in this way from $\mathbf{Z}_{2}$ twists have simple representations constructed in terms of $\gamma$-matrices (see e.g. [17] for an explicit construction). For example, a $\mathbf{Z}_{2}$ twist of the $S U(3)$ root lattice gives rise to an extra-special group that has a two-dimensional representation given by the conventional Pauli $\sigma$-matrices. These facts inspired FLM to consider a $\mathbf{Z}_{2}$ twisted version of the Leech theory in order to remove the unwanted 24 states that contribute to the constant term in the partition function, and to obtain the appropriate finite group structure. From an orbifold point of view, the centralizer of an involution 
in the monster has a very suggestive form. The group $\cdot 1$ can be regarded as the automorphism group of the Leech lattice with lattice elements identified with their negatives (i.e. after identifying $x \rightarrow-x$ ), and, as seen in the last section, $2^{12}$ is the degeneracy factor required in the twisted sector for a purely left-acting $\mathbf{Z}_{2}$ twist of 24 coordinates. The involution $i$ itself will turn out to be the symmetry of the operator algebra of a $\mathbf{Z}_{2}$ orbifold in which untwisted (twisted) states are assigned $+1(-1)$.

We now construct explicitly the $\mathbf{Z}_{2}$ twisted version of an arbitrary $c=24$ holomorphic conformal theory based on $\mathbf{R}^{24} / \Lambda$, where $\Lambda$ could be any of the 24 even, self-dual, Niemeier lattices in 24 dimensions. The conformal field theory of interest for the monster will then be given by the choice $\Lambda=\Lambda_{\text {Leech. }}$ With $g: x \rightarrow-x$, the untwisted Hilbert space $H_{(+)}$decomposes into $g= \pm 1$ eigenspaces as

$$
\begin{aligned}
& H_{(+)}^{+}=\left\{\alpha_{-n_{1}}^{i_{1}} \cdots \alpha_{-n_{2 k}}^{i_{2 k}}(|\beta\rangle+|-\beta\rangle)\right\}+\left\{\alpha_{-n_{1}}^{i_{1}} \cdots \alpha_{-n_{2 k+1}}^{i_{2 k+1}+1}(|\beta\rangle-|-\beta\rangle)\right\}, \\
& H_{(+)}^{-}=\left\{\alpha_{-n_{1}}^{i_{1}} \cdots \alpha_{-n_{2 k+1}}^{i_{2 k+1}}(|\beta\rangle+|-\beta\rangle)\right\}+\left\{\alpha_{-n_{1}}^{i_{1}} \cdots \alpha_{-n_{2 k}}^{i_{2 k}}(|\beta\rangle-|-\beta\rangle)\right\}
\end{aligned}
$$

$\left(n_{i} \in \mathbf{Z}, \beta \in \Lambda\right)$, and we find

$$
\begin{aligned}
& Z_{(+)}^{+}(q)=q^{-1} \operatorname{Tr}_{H_{(+)}^{+}} q^{L_{0}}=\frac{1}{2}\left(\frac{\Theta_{\Lambda}(q)}{\eta^{24}}+\frac{q^{-1}}{\prod_{n=1}^{\infty}\left(1+q^{n}\right)^{24}}\right), \\
& Z_{(+)}^{-}(q)=q^{-1} \operatorname{Tr}_{H_{(+)}^{-}} q^{L_{0}}=\frac{1}{2}\left(\frac{\Theta_{\Lambda}(q)}{\eta^{24}}-\frac{q^{-1}}{\prod_{n=1}^{\infty}\left(1+q^{n}\right)^{24}}\right) .
\end{aligned}
$$

The twisted Hilbert space $H_{(-)}$decomposes as

$$
\begin{aligned}
& H_{(-)}^{+}=\left\{\alpha_{-n_{1}}^{i_{1}} \cdots \alpha_{-n_{2 k+1}}^{i_{2 k+1}}|0\rangle \otimes\left|\sigma^{a}\right\rangle\right\}, \\
& H_{(-)}^{-}=\left\{\alpha_{-n_{1}}^{i_{1}} \cdots \alpha_{-n_{2 k}}^{i_{2 k}}|0\rangle \otimes\left|\sigma^{a}\right\rangle\right\}
\end{aligned}
$$

$\left(n_{i} \in \mathbf{Z}+\frac{1}{2},\left|\sigma^{a}\right\rangle \in T\right)$, so that

$$
\begin{aligned}
& Z_{(-)}^{+}(q)=q^{-1} \operatorname{Tr}_{H_{(-)}^{+}} q^{L_{0}}=q^{1 / 2} 2^{12} \frac{1}{2}\left(\frac{1}{\prod_{n=1}^{\infty}\left(1-q^{n-1 / 2}\right)^{24}}-\frac{1}{\prod_{n=1}^{\infty}\left(1+q^{n-1 / 2}\right)^{24}}\right), \\
& Z_{(-)}^{-}(q)=q^{-1} \operatorname{Tr}_{H_{(-)}^{-}} q^{L_{0}}=q^{1 / 2} 2^{12} \frac{1}{2}\left(\frac{1}{\prod_{n=1}^{\infty}\left(1-q^{n-1 / 2}\right)^{24}}+\frac{1}{\prod_{n=1}^{\infty}\left(1+q^{n-1 / 2}\right)^{24}}\right) .
\end{aligned}
$$

The partition function for the twisted theory is thus given by

$$
\begin{aligned}
Z_{\mathrm{tw}}(q) & =Z_{(+)}^{+}(q)+Z_{(-)}^{+}(q)=\frac{1}{2} \frac{\Theta_{\Lambda}(q)}{\eta^{24}}+\frac{1}{2} 2^{12}\left[\left(\frac{\eta}{\vartheta_{2}}\right)^{12}+\left(\frac{\eta}{\vartheta_{4}}\right)^{12}-\left(\frac{\eta}{\vartheta_{3}}\right)^{12}\right] \\
& =\frac{1}{2} \frac{\Theta_{\Lambda}(q)}{\eta^{24}}+\frac{1}{2} \frac{1}{\eta^{24}}\left[\left(\vartheta_{3} \vartheta_{4}\right)^{12}+\left(\vartheta_{2} \vartheta_{3}\right)^{12}-\left(\vartheta_{2} \vartheta_{4}\right)^{12}\right] \\
& =\frac{1}{2}(J(q)+24(h+1))+\frac{1}{2}(J(q)-24)=J+12 h,
\end{aligned}
$$


where $h=\left|\Lambda_{2}\right| / 24$ is the Coxeter number of the lattice, and $\vartheta_{i}=\vartheta_{i}(0, \tau)$ are the usual Jacobi theta-functions. The last line of (5.5) follows from the fact that $Z_{\mathrm{tw}}$ is a modular invariant function with a single pole with unit residue at $q=0$ (the cusp $\tau=i \infty$ ) and otherwise holomorphic, and it must therefore equal the modular function $J$ up to an additive constant. For $\Lambda=\Lambda_{\text {Leech }}$ as considered in (4.11), we have $\Lambda_{2}=\emptyset$, and (5.5) gives

$$
Z_{\text {Leech }}^{\mathrm{tw}}(q)=Z_{\text {Leech }}(q)-24=J(q) .
$$

We see that the twisting procedure preserves modular invariance while removing the 24 unwanted states $\alpha_{-1}^{i}|0\rangle$.

The twisted theory contains 196884 dimension 2 operators, including the stress tensor $T=-\frac{1}{2} \partial x \cdot \partial x$. In the untwisted Hilbert space there are $24 \cdot 25 / 2$ operators $\partial x^{i} \partial x^{j}$ and $196560 / 2$ more dimension 2 operators $\left(e^{i p \cdot x}+e^{-i p \cdot x}\right) / \sqrt{2}\left(p \in \Lambda_{4}\right)$. The remaining dimension 2 operators are the $24 \cdot 2^{12}$ operators $\tau^{i a}$ (see (4.24)) that create states $\alpha_{-1 / 2}^{i}\left|\sigma^{a}\right\rangle$ in the twisted Hilbert space. FLM define a closed algebra in terms of the Fourier components $\phi_{n}$, of these dimension 2 fields by defining a "cross-bracket,"

$$
\phi_{m}^{i} \times \phi_{n}^{j}=\frac{1}{2}\left(\left[\phi_{m+1}^{i}, \phi_{n-1}^{j}\right]+\left[\phi_{n+1}^{j}, \phi_{m-1}^{i}\right]\right),
$$

where $i, j$ label different dimension 2 fields. They then show that this cross bracket of dimension 2 fields closes on the Fourier components of other dimension 2 fields. This gives a commutative, non-associative, vertex operator algebra only slightly similar to the more familiar affine Lie algebras constructed from fields of dimension 1.

The above construction is easily understood from the OPE point of view. We have a conformal field theory with no dimension 1 fields, primary fields $\phi^{i}$ of dimension 2, a stress tensor $T(z)$ which is a descendant of the identity operator, and some set of primary dimension 3 fields $\chi^{i}$. The OPE of $\phi^{i}(z)$ with $\phi^{j}(w)$ then has the general form

$$
\begin{aligned}
\phi^{i}(z) \phi^{j}(w) \sim & \frac{1}{(z-w)^{4}} \delta^{i j}+\frac{1}{(z-w)^{2}}\left(b^{i j k} \phi^{k}(w)+\frac{4}{c} \delta^{i j} T(w)\right) \\
& +\frac{1}{(z-w)}\left(c^{i j k} \chi^{k}(w)+\frac{1}{2} \partial_{w}\left(b^{i j k} \phi^{k}(w)+\frac{4}{c} \delta^{i j} T(w)\right)\right)+\cdots,
\end{aligned}
$$

where Bose statistics requires symmetry of the coefficients $b^{i j k}$ in the first two indices $i, j$, and antisymmetry of the $c^{i j k}$ in $i, j$. The commutators in (5.7) can be extracted as usual from the singular terms in the OPE (5.8). In the cross bracket, the terms involving $\chi^{i}$ then cancel due to antisymmetry of the $c^{i j k}$.

Specializing to the case $c=24$, we obtain (using also the OPE's (2.3) and (2.4))

$$
\begin{aligned}
& \phi_{m}^{i} \times \phi_{n}^{j}=b^{i j k} \phi_{m+n}^{k}+\frac{1}{6} \delta^{i j} L_{m+n}+\frac{m^{2}}{2} \delta^{i j} \delta_{m+n, 0}, \\
& L_{m} \times \phi_{m}^{i}=2 \phi_{m+n}^{i}, \\
& L_{m} \times L_{n}=2 L_{m+n}+6 m^{2} \delta_{m+n, 0} .
\end{aligned}
$$


FLM have shown that the infinite dimensional closed algebra defined by this "cross-bracket" gives an affinization of the Griess algebra $B$, which appears as the dimension 196884 zero mode subalgebra (i.e. represented by the $\phi_{0}^{i}$ 's). The automorphism group of the Griess algebra is known to be precisely the monster [19]. From a "physical" point of view, the monster acts on this conformal field theory as a symmetry, commuting with the Hamiltonian and leaving the operator product coefficients invariant. The affinization of the Griess algebra is an example of a rather enlarged chiral algebra.

To give a better feeling for the monster, we recall that it can be generated by the centralizer $C$ of the involution $i$ together with another involution denoted $\sigma$ [19]. As mentioned at the beginning of this section, the involution $i$ is here the generic $\mathbf{Z}_{2}$ orbifold symmetry operation that acts as +1 on untwisted and -1 on twisted states. Because the group $C$ commutes with $i$, each of its elements takes untwisted to untwisted states, and twisted to twisted states. The action of the additional symmetry $\sigma$, on the other hand, exchanges twisted and untwisted states. To identify the symmetry $\sigma$ in our language, it is useful to regard the monster conformal field theory not as a $\mathbf{Z}_{2}$ orbifold of the conformal field theory based on the Leech lattice, but rather as a $\mathbf{Z}_{2} \times \mathbf{Z}_{2}$ orbifold of the conformal field theory based on the Niemeier lattice $\Lambda_{A_{1}^{24}}$. (This is a lattice constructed by adjoining to the $S U(2)^{24}$ root lattice additional weight vectors specified by the Golay code (see e.g. [20]).) The two elements generating the $\mathbf{Z}_{2} \times \mathbf{Z}_{2}$ group are $g: x \rightarrow-x$ and

$$
h: x \rightarrow x+2 \pi \cdot \frac{1}{4} \sum_{i=1}^{24} \alpha_{i}
$$

where $\alpha_{i}$ are the 24 positive roots of $S U(2)^{24}$. The $\mathbf{Z}_{2} \times \mathbf{Z}_{2}$ orbifold can be constructed in two steps, first modding out by the $\mathbf{Z}_{2}$ generated by the translation $h$, and then by the $\mathbf{Z}_{2}$ generating by $g$. The first step simply changes the lattice on which $x(z)$ is defined from $\Lambda_{A_{1}^{24}}$ to $\Lambda_{\text {Leech }}[1]$, and the second by definition gives the monster conformal field theory.

Acting directly on the $\Lambda_{A_{1}^{24}}$ theory, on the other hand, $g, h$, and $g h$ are all equivalent as $\mathbf{Z}_{2}$ twists. This may be seen by considering their actions on the level one $S U(2)^{24}$ current algebra of the theory,

$$
\begin{aligned}
& g: J_{1}^{i} \rightarrow+J_{1}^{i}, \quad J_{2}^{i} \rightarrow-J_{2}^{i}, \quad J_{3}^{i} \rightarrow-J_{3}^{i}, \\
& h: J_{1}^{i} \rightarrow-J_{1}^{i}, \quad J_{2}^{i} \rightarrow-J_{2}^{i}, \quad J_{3}^{i} \rightarrow+J_{3}^{i} \text {, } \\
& g h: J_{1}^{i} \rightarrow-J_{1}^{i}, \quad J_{2}^{i} \rightarrow+J_{2}^{i}, \quad J_{3}^{i} \rightarrow-J_{3}^{i},
\end{aligned}
$$

$(i=1, \ldots, 24)$ where

$$
J_{1}^{i}=\frac{1}{\sqrt{2}}\left(e^{i \sqrt{2} x^{i}}+e^{-i \sqrt{2} x^{i}}\right), \quad J_{2}^{i}=\frac{i}{\sqrt{2}}\left(e^{i \sqrt{2} x^{i}}-e^{-i \sqrt{2} x^{i}}\right), \quad J_{3}^{i}=i \partial x^{i}
$$

represent the currents. The three symmetry actions in (5.11) are equivalent due to the $S_{3}$ permutation symmetry of $S U(2)$ that interchanges the 3 currents. This triality symmetry is a symmetry of the full theory due to the $S U(2)^{24}$ symmetry. Because $g, h$ and $g h$ have equivalent $\mathbf{Z}_{2}$ actions, the $\mathbf{Z}_{2} \times \mathbf{Z}_{2}$ orbifold conformal field theory also has an $S_{3}$ permutation symmetry that permutes fields in the 3 twisted sectors 
$H_{g}, H_{h}$, and $H_{g h}$ among one another, and also permutes fields within the untwisted sector $H_{1}$. The (odd) permutation that exchanges $g$ and $h$ is the symmetry $\sigma$ that together with $C$ generates the monster.

\section{Superconformal Symmetry in the Moonshine Module}

It is now straightforward to identify the superconformal symmetry in the FLM construction. The direct sum of the untwisted and twisted Hilbert spaces, $H_{(+)} \oplus H_{(-)}$, before $g$ projection, gives rise to a $\mathbf{Z}_{2}$ non-local covering theory that contains $2^{12}$ primary dimension $3 / 2$ twist fields $\sigma^{a}$. In general, the $2^{12}$ states $\left|\sigma^{a}\right\rangle$ (belonging to $T$, as discussed after (4.16)) are linear combinations of the $2^{24}$ states $\left|\tilde{q}_{b}\right\rangle$ located at fixed points. However, it is always possible [16] to take one of the states, say $\left|\sigma^{0}\right\rangle$, to be located at any particular fixed point, say the origin. The remaining linear combinations of states $\left|\tilde{q}_{b}\right\rangle$ are then generated by taking operator products of the distinguished twist field $\sigma^{0}$ with all the $\mathbf{Z}_{2}$-invariant combinations of exponentials (along with their cocycles) from the untwisted sector. We tentatively identify $\sigma^{0}$ (up to normalization) with the field $T_{F}$ of a superconformal field theory. In order to check that (3.1) is satisfied, we need to determine the OPE of $\sigma^{0}(z)$ with $\sigma^{0}(w)$. Fields $\mathcal{O}(w)$ appearing in this OPE must create states in the untwisted Hilbert space and must have non-vanishing three-point function $\left\langle\sigma^{0}(z) \mathcal{O}(y) \sigma^{0}(w)\right\rangle \neq 0$. These three-point functions are determined by $S L(2, \mathbf{R})$ invariance, up to an overall factor that can be determined by calculating the expectation value $\left\langle\sigma^{0}|\mathcal{O}| \sigma^{0}\right\rangle$ of $\mathcal{O}(y)$ between twisted ground states $\left\langle\sigma^{0}\right|=\langle 0| \sigma^{0}(\infty)$ and $\left|\sigma^{0}\right\rangle=\sigma^{0}(0)|0\rangle$.

The space group selection rules [21] show that the only exponentials $e^{i \beta \cdot x(z)}$ appearing in the OPE of $\sigma^{0}(z)$ with $\sigma^{0}(w)$ have $\beta \in 2 \Lambda$, and none of these has dimension 2 (required for a singular contribution to the OPE). The three-point functions needed to determine the coefficient of $\partial x^{i} \partial x^{j}$ in this OPE can be easily calculated using the expansion (4.16), with the result that this operator appears only for $i=j$, and with coefficient independent of $j$. Thus the only dimension 2 operator that has a non-vanishing expectation value between the same twisted ground state is the stress tensor $T=-\frac{1}{2} \sum_{i=1}^{24} \partial x^{i} \partial x^{i}$. The coefficient of $T$ in the OPE is determined by associativity of the T $\sigma \sigma$ OPE (given that $\sigma^{0}$ is primary with dimension $3 / 2$ and canonically normalized, as in $(4.26)$ ) to be $2 \cdot(3 / 2) / c=1 / 8$. We see that $T_{F}=2 \sigma_{0}$ satisfies (3.1) and can therefore serve as a supersymmetry generator.

We can now organize the primary fields into Ramond and Neveu-Schwarz sectors. Fields in the $\mathrm{R}$ sector have square-root type branch cuts in their OPE with $T_{F}$. From (4.24) we see that the Ramond fields are those that create states in $H_{(-)}^{+}$or $H_{(+)}^{-}$. Similarly, NS fields create states in $H_{(+)}^{+}$or $H_{(-)}^{-}$. The $\mathbf{Z}_{2}$ twist generator $g$ plays the role that would be played by $\hat{\Gamma}$ in a general superconformal theory, in that projection onto the $g$ invariant states $H_{(+)}^{+} \oplus H_{(-)}^{+}$gives a local, modular invariant theory. The 24 states $\alpha_{-1}^{i}|0\rangle \in H_{(+)}^{-}$created by the untwisted fields $\partial x^{i}$ (which are Ramond fields with respect to $T_{F}=2 \sigma^{0}$ ) are Ramond ground states with $L_{0}=\hat{c} / 16=1 . G_{0}$ pairs the $24 \cdot 2^{12}=98304(\hat{\Gamma}=1)$ dimension 2 Ramond 
fields $\tau^{i a}$ with the $196560 / 2+24=98304(\hat{\Gamma}=-1)$ fields $\left(e^{i p \cdot x}-e^{-i p \cdot x}\right) / i \sqrt{2}$ and $\partial^{2} x^{i}$. This pairing of Ramond states into pairs with opposite eigenvalue of $\hat{\Gamma}=g$ is easily verified at all higher levels. To see this note that $q^{-1} \operatorname{Tr}_{H_{(+)}^{-}} q^{L_{0}}-24=$ $q^{-1} \operatorname{Tr}_{H_{(-)}^{+}} q^{L_{0}}$, as follows from the identities implicit in (5.5) (in general we have $\left.Z_{(+)}^{-}(q)-Z_{(-)}^{+}(q)=12 h+24\right)$.

In the NS sector, on the other hand, superconformal invariance organizes the fields into primary superfields. The identity operator is a NS field of dimension 0 . It gives rise to a dimension $3 / 2$ descendant $G_{-3 / 2}|0\rangle$ and a dimension 2 descendant $L_{-2}|0\rangle$ (recall that the vacuum is annihilated by $G_{-1 / 2}$ and $L_{-1}$ ). The remaining $2^{12}-1$ of the dimension $3 / 2$ NS fields $\sigma^{a}$ are lowest components of primary superfields of dimension $3 / 2$. The highest components of these superfields are the descendants with respect to $G_{-1 / 2}$ of the lowest components, i.e. they correspond to the states $G_{-1 / 2}\left|\sigma^{a}\right\rangle$. Subtracting these out, we are left with $196560 / 2+300-2^{12}=94484$ primary superfields of dimension 2 .

In general, the NS primary superfields can be classified according to their dimension and the $\hat{\Gamma}$ eigenvalue of their lowest component. To count primary superfields we must subtract all the states that are descendants under the superconformal algebra. Since the identity operator is annihilated by $G_{-1 / 2}$ and $L_{-1}$, it must be treated specially. We first subtract from $Z_{(+)}^{+}$and $Z_{(-)}^{-}$the descendants of the identity with $\hat{\Gamma}$ eigenvalue +1 and -1 respectively. This yields

$$
\begin{aligned}
& Y_{(+)}^{+}(q)=Z_{(+)}^{+}(q)-\frac{\frac{1}{2}\left(\prod_{n=2}^{\infty}\left(1+q^{n-1 / 2}\right)+\prod_{n=2}^{\infty}\left(1-q^{n-1 / 2}\right)\right)}{\prod_{n=2}^{\infty}\left(1-q^{n}\right)}, \\
& Y_{(-)}^{-}(q)=Z_{(-)}^{-}(q)-\frac{\frac{1}{2}\left(\prod_{n=2}^{\infty}\left(1+q^{n-1 / 2}\right)-\prod_{n=2}^{\infty}\left(1-q^{n-1 / 2}\right)\right)}{\prod_{n=2}^{\infty}\left(1-q^{n}\right)} .
\end{aligned}
$$

We let

$$
P_{ \pm}(q)=q^{-1} \sum_{h>0} N_{ \pm}(h) q^{h}
$$

be the generating function for primary NS superfields (other than the identity) with $\hat{\Gamma}$ eigenvalue \pm 1 , where $N_{ \pm}(h)$ is the number of NS primary superfields of dimension $h$ and $\hat{\Gamma}$ eigenvalue \pm 1 . Writing $Y_{(+)}^{+}$and $Y_{(-)}^{-}$in terms of primary fields and their descendants and then solving for $P_{ \pm}$gives

$$
P_{ \pm}(q)=\frac{1}{2} \prod_{n=1}^{\infty}\left(1-q^{n}\right)\left[\frac{Y_{(+)}^{+}(q)+Y_{(-)}^{-}(q)}{\prod_{n=1}^{\infty}\left(1+q^{n-1 / 2}\right)} \pm \frac{Y_{(+)}^{+}(q)-Y_{(-)}^{-}(q)}{\prod_{n=1}^{\infty}\left(1-q^{n-1 / 2}\right)}\right] .
$$

While it is relatively easy to count the number of primary superfields at a given level, it is more difficult to determine precisely which combinations of fields correspond to these superfields. This involves a more detailed analysis of the structure of the Leech lattice and the two-cocycles $\varepsilon(\alpha, \beta)$ than will be attempted here. In 
any event we see how the numerology associated with the coefficients of the expansion of the modular invariant $J$ of (4.11) may be further refined by recognition of the superconformal symmetry in the FLM construction.

Now that we have identified the superconformal symmetry in the FLM construction, it is possible to give a superconformal interpretation of the removal of the 24 unwanted states from the Leech theory (4.11). Every superconformal theory has a $\mathbf{Z}_{2}$ symmetry that we denote $(-1)^{F_{s}}$, defined to act as +1 on states in the antiperiodic (NS) sector of the worldsheet supersymmetry generator, and as -1 on states in the periodic $(\mathrm{R})$ sector. (We refer to this symmetry as $(-1)^{F_{s}}$ since it resembles the action of an operator $(-1)^{F_{s}}$ in string theory, where $F_{s}$ would be the spacetime fermion number.) In Appendix A of [10], it was shown that modding out by this symmetry (i.e. take $g=(-1)^{F_{s}}$ in (4.23)) gives another superconformal theory with opposite sign for the term (3.5). (Modding out the resulting theory again by $(-1)^{F_{s}}$ gives back the original theory.) Modding out the twisted Leech theory by $(-1)^{F_{s}}$ thus gives back a superconformal theory in which the 24 Ramond ground states are restored. This theory is in fact the original Leech theory $Z_{\text {Leech }}$, which we now recognize to possess a superconformal symmetry as well. One way to see this is to recognize that the action of $(-1)^{F_{s}}$ on the states of the twisted Leech theory $\left(+1\right.$ on $H_{(+)}^{+}$and -1 on $H_{(-)}^{+}$) is identical to that of the $\mathbf{Z}_{2}$ symmetry that would generically undo the orbifolding by $x \rightarrow-x$ (i.e. the involution $i$, defined to act as +1 on the untwisted sector, and -1 on the twisted sector). In superconformal language, then, the modification that removes the 24 unwanted states from the Leech theory can be equally well taken to be the $\mathbf{Z}_{2}$ symmetry generated by $(-1)^{F_{s}}$, and the argument preceding (3.5) gives the natural explanation in this language for why the partition function is changed by a constant.

Having introduced the symmetry $(-1)^{F_{s}}$, we can now complete the discussion at the end of Sect. 3 concerning when a given conformal theory is superconformal. A key role in this identification is played by the $\mathbf{Z}_{2}$ symmetry $(-1)^{F}$, because it is a symmetry of any superconformal field theory (holomorphic or not). If a modular invariant superconformal theory is twisted by this $\mathbf{Z}_{2}$ symmetry, the operator $T_{F}$ appears in the twisted Hilbert space $H_{(-)}$(i.e. before projection onto $\mathbf{Z}_{2}$-invariant states - the final $\mathbf{Z}_{2}$ projection, giving a modular invariant partition function, would again eliminate $T_{F}$ ). To determine whether a conformal field theory is superconformal or not, one may thus twist by all $\mathbf{Z}_{2}$ symmetries of the operator algebra, and in each case look for a dimension $\left(\frac{3}{2}, 0\right)$ field in the twisted sector that obeys (3.1), and also has the appropriate local behavior with respect to the $\mathbf{Z}_{2}$-even (Neveu-Schwarz) fields and a square-root type non-local behavior with respect to the $\mathbf{Z}_{2}$-odd (Ramond) fields. If there is such a symmetry, then the combined untwisted plus twisted Hilbert space before $\mathbf{Z}_{2}$ projection, $H_{(+)} \oplus H_{(-)}$, provides the desired non-local $\mathbf{Z}_{2}$ covering theory. The $\mathbf{Z}_{2}$ symmetry that projects back onto the original modular invariant theory, now recognized as superconformal, is simply that assigning $+1(-1)$ to states in $H_{(+)}\left(H_{(-)}\right)$.

It is easy to see that the supersymmetry in the twisted Leech theory terminates at $N=1$. This is because higher supersymmetry algebras involve as well dimension one currents not present in the theory (5.6). The monster group, although an automorphism group of the full conformal field theory, does not preserve the 
supersymmetry generator, and the supersymmetric structure we have identified is invariant only under a subgroup (contained in the subgroup $C$ ) of the monster group.

\section{The Monster and Genus Zero Function Fields}

Perhaps the most mysterious element in the monstrous game is the relation between elements of the monster and genus zero function fields. In this section we will give a brief summary (again oriented towards string theorists) of what is known about this relation. A more detailed discussion can be found in [2,22].

The fundamental domain for the modular group, $\mathbf{H} / \operatorname{SL}(2, \mathbf{Z})$ (where $\mathbf{H}$ is the upper half-plane) is a familiar object to string theorists. After adjoining the point at infinity $(\tau=i \infty)$, this space can be given a topology and a complex structure so that it becomes a compact Riemann surface. The modular function $j$ provides a map of this compactification of $\mathbf{H} / S L(2, \mathbf{Z})$ to the Riemann sphere $\mathbf{C} \cup \infty$ which is one-to-one and onto. As a result, we see that the Riemann surface associated with $\mathbf{H} / S L(2, \mathbf{Z})$ has genus zero. It follows that the field $K_{S L(2, \mathbf{Z})}$ of meromorphic functions on this Riemann surface consists of all rational functions of $j$ with complex coefficients, i.e. any $k \in K_{S L(2, Z)}$ satisfies

$$
k=\frac{\sum_{i=0}^{n} a_{i} j^{i}}{\sum_{i=0}^{m} b_{i} j^{i}}
$$

with $a_{i}, b_{i} \in \mathbf{C} . K_{S L(2, Z)}$ is said to be a genus zero function field, and the modular function $j$ is the generator, or hauptmodul of this function field.

This discussion can be repeated for other discrete subgroups $\Gamma \subset S L(2, \mathbf{R})$, known as groups of genus zero, that have the property that the appropriate compactification of $\mathbf{H} / \Gamma$ yields a Riemann surface of genus zero. The field of rational functions on the compactification of $\mathbf{H} / \Gamma$ is a genus zero function field with generator $j_{\Gamma}$, analogous to the modular function $j$ in the case $\Gamma=S L(2, \mathbf{Z})$. Examples of such genus zero subgroups of $S L(2, \mathbf{R})$ are provided by the congruence subgroups $\Gamma_{0}(p)$, where $p$ is a prime such that $p-1$ divides 24 . Here $\Gamma_{0}(N)$ is defined as

$$
\Gamma_{0}(N)=\left\{\left(\begin{array}{ll}
a & b \\
c & d
\end{array}\right) \in S L(2, \mathbf{Z}) \mid c=0 \bmod N\right\} .
$$

Another group of interest, denoted $\Gamma_{0}(N)^{+}$, is obtained by adjoining the Fricke involution $\tau \rightarrow-1 / N \tau$ to $\Gamma_{0}(N){ }^{2}$ It was observed in [24] that the primes $p$ for which $\Gamma_{0}(p)^{+}$has genus zero are precisely the primes that divide the order of the monster!

Since the monster acts as a symmetry of the (super) conformal field theory constructed by FLM, it has a well-defined action on each graded subspace, and

\footnotetext{
${ }^{2}$ See [23] for an application of these groups in a string theory context
} 
one can define the series

$$
T_{g}(q)=q^{-1} \operatorname{Tr} g q^{L_{0}},
$$

where $g$ is an element of the monster, and the trace runs over all states in the theory. These series are known as Thompson-McKay series. Conway and Norton have conjectured that the Thompson-McKay series associated to each rational conjugacy class of the monster is a hauptmodul for a genus zero subgroup $\Gamma \subset S L(2, \mathbf{R})$ that contains $\Gamma_{0}(N)$ and is contained in the normalizer of $\Gamma_{0}(N)$ in $S L(2, \mathbf{R})$. (The normalizer of a subgroup $H \subset G$ consists of all elements $g \in G$ such that $g^{-1} h g \in H$ for all $h \in H$. A rational conjugacy class is one whose character is a rational number.)

The Thompson series $T_{g}(q)$ can be viewed as a path integral over the torus with modular parameter $\tau$ and boundary conditions given by $g \square$. In the spirit of orbifolds, it is natural to consider more general boundary conditions on the torus. If $g$ and $h$ are two commuting elements of the monster, then it makes sense to consider the path integral (4.21) with boundary conditions twisted by $g$ and $h$ in the "time" and "space" directions. This corresponds to the trace

$$
Z(g, h ; q) \equiv g \prod_{h}=q^{-1} \operatorname{Tr}_{H_{h}} g q^{L_{0}},
$$

evaluated in the Hilbert space twisted by $h$. The standard orbifold result that $Z(g, h ; q)$ is invariant under simultaneous conjugation of both $g$ and $h$, and the result that the boundary conditions (4.21) transform as in (4.22) under modular transformations, appear as parts one and three of the conjecture by Norton in the appendix of [22]. Norton also conjectures that these generalized ThompsonMcKay series are invariant under genus zero subgroups of $\operatorname{SL}(2, \mathbf{R})$. This conjecture strengthens our suspicion that holomorphic orbifold (super) conformal field theories may provide the proper setting for trying to obtain a conceptual understanding of the relations between the monster and genus zero function fields.

\section{Discussion}

It would be interesting to investigate other orbifold holomorphic conformal field theories in an attempt to generalize the FLM construction of the monster to other sporadic groups, and to study the structure of twisted vertex operator algebras. It is easy using (5.5) to check that $\mathbf{Z}_{2}$ twists of the other 23 Niemeier lattices lead in some cases to holomorphic conformal field theories which are not graded isomorphic to any of the untwisted theories based on Niemeier lattices. It might be illuminating to investigate the structure of the cross-bracket algebra in these cases as well. We point out that the arguments given in the second paragraph of Sect. 6, showing that the twist field is a supersymmetry generator, apply equally well to $\mathbf{Z}_{2}$ twisted versions of the $c_{i}=24$ holomorphic conformal field theories based on the remaining 23 Niemeier lattices. According to (5.5), the $x \rightarrow-x$ modification always removes $12 h+24$ states, which may then be restored by modding out the resulting superconformal theory by $(-1)^{F_{s}}$, thereby manifesting 
as well a superconformal invariance in the untwisted Niemeier theories. Since the Niemeier conformal theories in general possess spin one states in their spectra, we suspect the occurrence of higher $(N>1)$ superconformal algebras in many cases (depending on the detailed structure of the cocycles).

It is also possible that one may need to consider holomorphic conformal field theories invariant only under a subgroup of the modular group. In [1], for example, FLM provide some evidence that a twisted version of compactification of the left-moving superstring on the $E_{8}$ lattice also has moonshine properties for $\cdot 1$. This theory also has an underlying superconformal symmetry, but again it is not clear what role, if any, it plays in the moonshine.

The main outstanding problems related to the moonshine module are the understanding of the relation between elements of the monster and genus-zero function fields; and a generalization of the techniques reviewed here to other sporadic groups, particularly the "pariahs" which are not involved in the monster. We reiterate our hope that the techniques of (super) conformal field theory will play a useful role in the solution to these problems.

Acknowledgements. L. D. is supported by NSF contract PHY80-19754; P. G. by NSF contract PHY-82-15249, DOE OJI grant FG-84ER40171, and by the A. P. Sloan foundation; J. H. by NSF contract PHY80-19754, PYI award PHY86-58033, and by the A. P. Sloan foundation. We are grateful to I. Frenkel, J. Lepowsky, and G. Moore for comments on the manuscript, and to V. Kaplunovsky for a useful discussion.

\section{References}

1. Frenkel, I. B., Lepowsky, J., Meurman, A.: A natural representation of the Fischer-Griess monster with the modular function $J$ as character. Proc. Natl. Acad. Sci. U.S.A. 81, 32566 (1984); Frenkel, I. B., Lepowsky, J., Meurman, A.: A moonshine module for the monster. In: Vertex operators in mathematics and physics. Lepowsky, J., Mandelstam, S., Singer, I (eds.). Publ. Math. Sciences Res. Inst. Vol. 3, p. 231. Berlin, Heidelberg, New York: Springer 1985; Frenkel, I. B., Lepowsky, J., Meurman, A.: Vertex operator algebras and the monster. New York: Academic Press (to appear)

2. Conway, J. H., Norton, S. P.: Monstrous moonshine. Bull. Lond. Math. Soc. 11, 308 (1979)

3. Belavin, A. A., Polyakov, A. M., Zamolodchikov, A. B.: Infinite conformal symmetry in twodimensional quantum field theory. Nucl. Phys. B241, 333 (1984); Friedan, D.: Notes on string theory and two dimensional conformal field theory. In: Unified string theories. Green, M., Gross, D. (eds.). Singapore: World Scientific 1986; Peskin, M.: Introduction to string and superstring theory II. 1986 TASI lectures, SLAC-PUB-4251; Banks, T.: Lectures on conformal field theory. 1987 TASI lectures, SCIPP 87/111

4. Friedan, D., Qiu, Z., Shenker, S.: Conformal invariance, unitarity, and critical exponents in two dimensions. Phys. Rev. Lett. 52, 1575 (1984)

5. Cardy, J.: Operator content of two-dimensional conformally invariant theories. Nucl. Phys. B270[FS16], 186 (1986)

6. Moore, G.: private communication

7. Gliozzi, F., Scherk, J., Olive, D.: Supersymmetry, supergravity theories and the dual spinor model. Nucl. Phys. B122, 253 (1977); Friedan, D., Qiu, Z., Shenker, S.: Superconformal invariance in two dimensions and the tricritical Ising model. Phys. Lett. 151B, 37 (1985)

8. Witten, E.: Constraints on supersymmetry breaking. Nucl. Phys. B202, 253 (1982)

9. Kastor, D.: Modular invariance in superconformal models. Nucl. Phys. B280[FS18] 304 (1987)

10. Dixon, L., Ginsparg, P., Harvey, J.: $\hat{c}=1$ superconformal field theory. Nucl. Phys. B306, 470 (1988) 
11. Narain, K. S., Sarmadi, M. H., Witten, E.: A note on toroidal compactification of heterotic string theory. Nucl. Phys. B279 369 (1986); Ginsparg, P.: On toroidal compactification of heterotic superstrings. Phys. Rev. D35, 648 (1987)

12. Frenkel, I. B., Kac, V. G.: Basic representations of affine Lie algebras and dual resonance models. Invent. Math. 62, 23 (1980); Segal, G.: Unitary representations of some infinite dimensional groups. Commun. Math. Phys. 81, 301 (1981)

13. Dixon, L., Harvey, J., Vafa, C., Witten, E.: Strings on orbifolds I, II. Nucl. Phys. B261, 678 (1985); Nucl. Phys. B274 285 (1986)

14. Vafa, C.: Modular invariance and discrete torsion on orbifolds. Nucl. Phys. B273, 592 (1986)

15. Lepowsky, J.: Calculus of twisted vertex operators. Proc. Natl. Acad. Sci. USA 82, 8295 (1985)

16. Narain, K. S., Sarmadi, M. H., Vafa, C.: Asymmetric orbifolds. Nucl. Phys. B288, 551 (1987)

17. Corrigan, E., Hollowood, T.: Comments on the algebra of straight, twisted, and intertwining vertex operators. Nucl. Phys. B304, 77 (1988)

18. Tits, J.: Normalisateurs de tores 1. Groups de Coxeter étendus. J. Alg. 4, 96 (1966)

19. Griess, R. L., Jr.,: The friendly giant. Invent. Math. 69, 1 (1982); Tits, J.: On R. Griess' 'Friendly Giant'. Invent. Math. 78, 491 (1984)

20. Conway, J. H., Sloane, N. J. A.: Twenty-three constructions for the Leech lattice. Proc. Roy. Soc. Lond. Ser. A381, 275 (1982)

21. Dixon, L., Friedan, D., Martinec, E., Shenker, S.: The conformal field theory of orbifolds. Nucl. Phys. B282, 13 (1987); Hamidi, S., Vafa, C.: Interactions on orbifolds. Nucl. Phys. B279, 465 (1987)

22. Mason G. (with an appendix by Norton, S. P.);: Finite groups and modular functions. Proc. Symposia in Pure Math. 47, 181 (1987)

23. Moore, G.: Atkin-Lehner symmetry. Nucl. Phys. B293, 139 (1987). Erratum ibid. B299, 847 (1988)

24. Ogg, A.: Hyperelliptic modular curves. Bull. Soc. Math. France 102, 449 (1974)

Communicated by L. Alvarez-Gaumé

Received April 27, 1988 
